ARTICLE

Received 17 Aug 2012 | Accepted 25 Mar 2013 | Published 30 Apr $2013 \quad$ DOl: 10.1038/ncomms2806

\title{
Active cochlear amplification is dependent on supporting cell gap junctions
}

Yan Zhu ${ }^{1, \star}$, Chun Liang ${ }^{1,2, \star}$, Jin Chen ${ }^{1,3, \star}$, Liang Zong ${ }^{1}$, Guang-Di Chen ${ }^{4} \&$ Hong-Bo Zhao ${ }^{1}$

Mammalian hearing relies upon active cochlear mechanics, which arises from outer hair cell electromotility and hair bundle movement, to amplify acoustic stimulations increasing hearing sensitivity and frequency selectivity. Here we describe the novel finding that gap junctions between cochlear supporting cells also have a critical role in active cochlear amplification in vivo. We find that targeted-deletion of connexin 26 in Deiters cells and outer pillar cells, which constrain outer hair cells standing on the basilar membrane, causes a leftward shift in outer hair cell electromotility towards hyperpolarization, and reduces active cochlear amplification with hearing loss. Coincident with large reduction in distortion product otoacoustic emission and severe hearing loss at high frequencies, the shift is larger in shorter outer hair cells. Our study demonstrates that active cochlear amplification in vivo is dependent on supporting cell gap junctions. These new findings also show that connexin 26 deficiency can reduce active cochlear amplification to induce hearing loss.

\footnotetext{
${ }^{1}$ Department of Otolaryngology, University of Kentucky Medical School, Lexington, Kentucky 40536, USA. ${ }^{2}$ Maternal and Child Health Care Hospital of Baoan, Shenzhen 518133, PRC. ${ }^{3}$ Department of Otolaryngology, Tongji Hospital, Huazhong University of Science and Technology, Wuhan 430030, PRC.

${ }^{4}$ Center for Hearing \& Deafness, SUNY, Buffalo, New York 14214, USA. * These authors contributed equally to this work. Correspondence and requests for materials should be addressed to H.-B.Z. (email: hzhao2@uky.edu).
} 
$\mathrm{M}$ ammalian hearing function relies upon active cochlear amplification amplifying the basilar membrane (BM) vibration to acoustic stimulation increasing hearing sensitivity and frequency selectivity ${ }^{1,2}$. Outer hair cell $(\mathrm{OHC})$ electromotility is an active cochlear amplifier in mammals $s^{3-6}$. Deficiency of OHC electromotility can induce hearing $\operatorname{loss}^{7}$. In situ, OHCs are constrained by phalangeal processes of Deiters cells (DCs) and pillar cells (PCs). They contain rigid bundles of microtubules and actin microfilaments along their phalangeal processes acting as a scaffold supporting OHCs standing between the $\mathrm{BM}$ and the reticular lamina ${ }^{8,9}$. This rigid scaffold also transfers forces between the $\mathrm{BM}$ and the reticular lamina and provides an appropriate mechanical load to the OHCs to maintain OHC electromotility at an optimal operating point.

It has been found that the phalangeal processes of DCs can alter their curvature in responses to electrical and chemical stimulations ${ }^{10-12}$. However, DCs do not have OHC-like electromotility. DCs and PCs including their phalangeal processes are well coupled by gap junctions but hair cells have neither gap junctions nor connexin expression ${ }^{6,13-15}$. There is also no direct electrical conductance between DCs and OHCs ${ }^{15,16}$. We previously found that electrical and mechanical stimulations in DCs or alternation of gap junctions between DCs can affect OHC electromotility in the in vitro preparation ${ }^{16}$. However, it remains unclear whether supporting cell gap junctions in vivo can also alter active cochlear mechanics to influence hearing.

Connexin 26 (Cx26, GJB2) is a predominant gap junction isoform in the cochlea ${ }^{13,14,17}$. In this study, we selectively deleted Cx26 expression in DCs and outer pillar cells (OPCs), which directly constrain OHCs standing on the BM. We found that targeted-deletion of Cx26 in DCs and OPCs left-shifted OHC electromotility and reduced active cochlear mechanics with hearing loss. These data demonstrate that $\mathrm{Cx} 26$ expression in supporting cells has a critical role in active cochlear amplification in vivo. In the clinic, Cx26 mutations produce a high incidence of hearing loss and are responsible for $\sim 50 \%$ of nonsyndromic hearing loss ${ }^{18-20}$. We recently found that cell degeneration is not a primary cause for deafness due to $\mathrm{Cx} 26$ deficiency $^{21}$. These new findings also reveal a new mechanism for Cx26 deficiencyassociated hearing loss.

\section{Results}

Targeted-deletion of Cx26 in DCs and OPCs in the cochlea. We used loxP-Cre technique to delete Cx26 expression in DCs and OPCs. The Cx26 conditional knockout (cKO) mouse was generated by crossing $C \times 26^{\text {loxP/loxP }}$ transgenic mice ${ }^{22}$ with a Proxi-CreER ${ }^{T 2}$ mouse line, which a tamoxifen-inducible Cre recombinase is driven by a homeodomain transcription factor Prox1 (ref. 23). Prox1 is specifically expressed in DCs and OPCs in the cochlea after E18 and remains in these cells after birth ${ }^{24-26}$. Consistent with Cre expression ${ }^{26}$, Cx26 labelling in DCs and OPCs was absent in Cx26 cKO mice (Fig. 1, Supplementary Figs S1 and S2). Cx26 labelling in the inner layer of Hensen cells was also reduced but not absent (Fig. 1b,c,g,h). The deletion is uniform at apical, middle and basal turns (Supplementary Fig. S1). Cx26 labelling in other cochlear structures including the spiral limbus (SLM) and the lateral wall was intense and normal (Fig. 1a-e and Supplementary Fig. S2). Cx30 is another predominant gap junction isoform expressed in the cochlea ${ }^{14,17}$. Cx30 expression also appeared normal in Cx26 cKO mice. Intense labelling for $\mathrm{Cx} 30$ in DCs and OPCs is visible (Fig. 1c,h and Supplementary Fig. S2). In addition, the cochlea appeared normal development in Cx26 cKO mice (Fig. 1e and Supplementary Fig. S1).
Elevation of auditory brainstem response threshold. The $\mathrm{Cx} 26$ cKO mice had hearing loss (Fig. 2). The auditory brainstem response (ABR) threshold in homozygous $\mathrm{Cx} 26$ cKO mice was increased significantly. The thresholds at $8,16,24,32$ and $40 \mathrm{kHz}$ were $59.9 \pm 5.05,52.0 \pm 7.67,73.8 \pm 8.64,86.9 \pm 8.16$ and $93.4 \pm 4.58 \mathrm{~dB}$ SPL, respectively (Fig. $2 c, P<0.001$, one-way analysis of variance (ANOVA) with a Bonferroni correction). In comparison with wild-type (WT) littermates, the increase of ABR threshold in Cx26 cKO mice at 8, 16, 24, 32 and $40 \mathrm{kHz}$ was $15.3 \pm 5.05,16.7 \pm 7.67,38.5 \pm 8.64,45.7 \pm 8.16$ and $46.9 \pm 4.58 \mathrm{~dB}$ SPL, respectively (Fig. 2d). The increase at high frequencies was larger. The thresholds were elevated by $30-45 \mathrm{~dB}$ SPL at frequencies above $24 \mathrm{kHz}$. The ABR threshold was also increased in heterozygous Cx26 cKO mice (Fig. 2c,d). However, the increases of ABR threshold were less than those in homozygous mice. The ABR thresholds at 24,32 and $40 \mathrm{kHz}$ were $51.8 \pm 5.73,72.5 \pm 6.18$ and $77.1 \pm 7.35 \mathrm{~dB}$ SPL, respectively $(P<0.001$, one-way ANOVA with a Bonferroni correction). The threshold was increased by $15-30 \mathrm{~dB}$ at frequencies above $24 \mathrm{kHz}$ (Fig. 2d).

Reduction of distortion product otoacoustic emission. Figure 3 shows that targeted-deletion of Cx26 in DCs and OPCs significantly reduced distortion product otoacoustic emission (DPOAE), which reflects activity of active cochlear amplification in vivo. The distortion product of $2 f_{1}-f_{2}$ at $f_{0}$ of $20 \mathrm{kHz}$ was almost at the noise level and was $6.92 \pm 6.37,4.47 \pm 3.43$ and $11.9 \pm 7.89 \mathrm{~dB}$ SPL at the stimulus level of 40,50 and $60 \mathrm{~dB}$ SPL, respectively (Fig. 3a,c). In comparison with WT littermates, the DPOAEs were reduced by $15.3 \pm 6.92, \quad 30.2 \pm 4.47$ and $30.9 \pm 7.89 \mathrm{~dB}$ at the stimulus level of 40,50 and $60 \mathrm{~dB} \mathrm{SPL}$, respectively $(P<0.001$, one-way ANOVA with a Bonferroni correction). The decrease was larger at higher stimulus levels (Fig. 3d). The decrease was also larger at higher frequencies (Fig. 3b). The DPOAEs at 16 and $20 \mathrm{kHz}$ were reduced by $15.8 \pm 7.23$ and $30.9 \pm 7.89 \mathrm{~dB}$, respectively $(P<0.001$, one-way ANOVA with a Bonferroni correction). The DPOAE at $8 \mathrm{kHz}$ in $\mathrm{Cx} 26 \mathrm{cKO}$ mice was also reduced by $3.69 \pm 4.61 \mathrm{~dB}$. However, the decrease was not significant $(P=0.13$, one-way ANOVA). The DPOAE in heterozygous Cx26 cKO mice was also reduced (Fig. 3b-d). The decrease at the stimulus level of 50 and $60 \mathrm{~dB}$ SPL was about $10 \mathrm{~dB}$ SPL $(P<0.001$, one-way ANOVA with a Bonferroni correction), which was less than that in homozygous Cx26 cKO mice (Fig. 3c,d). The decrease was also larger at high frequencies (Fig. 3b). The decrease at 16 and $20 \mathrm{kHz}$ in the heterozygous mice was $8.95 \pm 7.01$ and $13.3 \pm 7.19 \mathrm{~dB}$, respectively $(P<0.001$, one-way ANOVA with a Bonferroni correction).

No apparent cell degeneration in the $\mathrm{Cx} 26$ cKO mouse cochlea. Targeted-deletion of Cx26 in DCs and OPCs did not cause apparent hair cell loss and cell degeneration in the inner ear. Figure 4a shows that hair cell loss in Cx26 cKO mice was $<5 \%$ at P30-60 and was not significantly different from WT mice. In particular, there was no signification hair cell loss at the highfrequency region $(P>0.05$, one-way ANOVA). Spiral ganglion (SG) neurons also had no apparent degeneration in Cx26 cKO mice (Fig. 5). In comparison with WT littermates, the density of SG neurons in Rosenthal's canal was not significantly reduced in Cx26 cKO mice $(P>0.05$, one-way ANOVA, Fig. $5 b)$.

Normal cochlear microphonics and endocochlear potentials. Cochlear microphonics $(\mathrm{CM})$ is the auditory receptor potential and is produced by hair cell mechanoelectrical transduction channel activity and endocochlear potential (EP) driving force. $\mathrm{CM}$ in $\mathrm{Cx} 26 \mathrm{cKO}$ mice was normal (Fig. 4b). Cx26 cKO mice also had normal endocochlear potential (EP). The recorded EP in WT, 

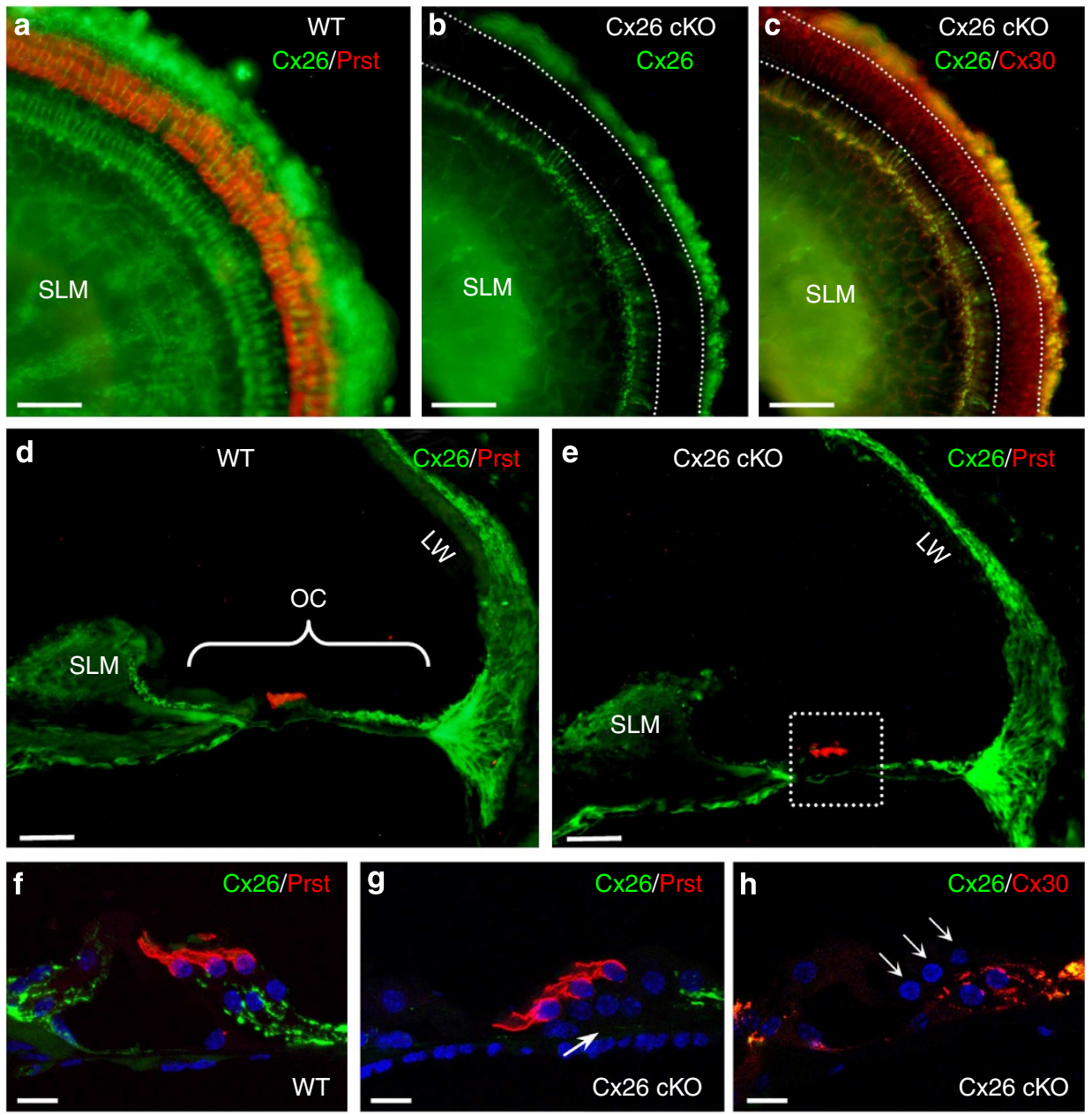

Figure 1 | Targeted-deletion of Cx26 in DCs and OPCs in Cx26 cKO mice. (a-c) Immunofluorescent labelling of the cochlear sensory epithelium for Cx26, Prestin and $\mathrm{C} \times 30$ in the whole-mounting preparation. White dashed lines represent OHC, DC and OPC area, where Cx26 labelling (green) is absent but Cx30 labelling (red colour in c) remains in Cx26 cKO mice. (d,e) Immunofluorescent staining of the cochlear cross-section for Cx26. OHCs are visualized by prestin labelling (red colour). A white dashed line box in e indicates OHCs, DCs, OPCs and their neighbouring area. OC, organ of Corti; LW, lateral wall; SLM, spiral limbus. (f-h) High-magnification images of the boxed area. A white arrow in $\mathbf{g}$ indicates that the Cx26 labelling in the DC and OPC region is absent in Cx26 cKO mice. Three white arrows in $\mathbf{h}$ indicate three rows of OHCs. Scale bars, $50 \mu \mathrm{m}$ in $\mathbf{a}-\mathbf{e}$; $10 \mu \mathrm{m}$ in $\mathbf{f}-\mathbf{h}$

heterozygous and homozygous mice was $92.0 \pm 2.31 \quad(n=4)$, $95.0 \pm 0.58(n=3)$ and $91.5 \pm 0.50 \mathrm{mV}(n=2)$, respectively.

Left-shift of OHC nonlinear capacitance in $\mathrm{Cx} 26 \mathrm{cKO}$ mice. $\mathrm{OHC}$ electromotility associated nonlinear capacitance (NLC) in $\mathrm{Cx} 26 \mathrm{cKO}$ mice retained a normal bell shape but was shifted to the left in the hyperpolarization direction (Fig. 6a). The voltage of peak capacitance $\left(V_{\mathrm{pk}}\right)$ was significantly shifted from $-73.8 \pm 2.14 \mathrm{mV}$ in WT mice to $-90.9 \pm 2.22 \mathrm{mV}$ in homozygous mice (Fig. 6b, $P=0.00004$, one-way ANOVA with a Bonferroni correction). $V_{\mathrm{pk}}$ in heterozygous mice was $-82.9 \pm 2.37 \mathrm{mV}$ and also had a significant left shift by $\sim 10 \mathrm{mV}(P=0.0003$, one-way ANOVA with a Bonferroni correction). The $V_{\mathrm{pk}}$ shifting is larger in shorter OHCs (Fig. 7). Linear regression analysis shows that the slope of $V_{\mathrm{pk}}$ shift with cell length is $2.33 \pm 0.55$ (mean \pm s.d., $r^{2}=0.22$ )

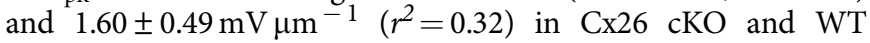
mice, respectively (Fig. 7a). The slopes had significant difference between $\mathrm{Cx} 26 \mathrm{cKO}$ and WT mice $(P=0.004$, Univariate analysis of variance). The $V_{\mathrm{pk}}$ at $\mathrm{OHC}$ lengths $<15,15-20$ and $>20 \mu \mathrm{m}$ was left-shifted by $-20.8 \pm 4.25,-14.6 \pm 2.72$ and $-10.37 \pm 3.81 \mathrm{mV}$, respectively, in $\mathrm{Cx} 26 \mathrm{cKO}$ mice (Fig. $7 \mathrm{~b}$, $P<0.05$, one-way ANOVA with a Bonferroni correction).
However, there was no significant difference in linear capacitance $\left(C_{\text {lin }}\right)$ (Fig. 6e). The $C_{\text {lin }}$ in homozygous, heterozygous and WT mice was $6.23 \pm 0.43,6.65 \pm 0.27$ and $6.73 \pm 0.34 \mathrm{pF}$, respectively $(P=0.84$, one-way ANOVA). Direct length measurement also shows that the average of lengths of recorded OHCs in WT and $\mathrm{Cx} 26 \mathrm{cKO}$ mice was $19.04 \pm 0.76(n=24)$ and $18.09 \pm 2.14 \mu \mathrm{m}$ $(n=68)$, respectively. There was no significant difference between them $(P=0.28, t$-test $)$.

No reduction in prestin expression and function. Maximum charge $\left(Q_{\max }\right)$ reflects the functional expression of prestin at the OHC lateral wall. $Q_{\max }$ in WT mice and heterozygous and homozygous $\mathrm{Cx} 26 \mathrm{cKO}$ mice was $0.72 \pm 0.04,0.75 \pm 0.04$ and $0.80 \pm 0.04 \mathrm{pC}$, respectively (Fig. 6c). The $Q_{\max }$ in $\mathrm{Cx} 26 \mathrm{cKO}$ mice had a slight increase rather than decrease. However, the increase was not significant $(P=0.13$, one-way ANOVA). Real-time RTPCR measurement also shows that the prestin expression at the transcription level was slightly increased rather than decreased in Cx26 cKO mice (Fig. 8). The increase was also not significant $(P=0.58$, one-way ANOVA). There was no difference in valence $z$ as well among WT mice, and heterozygous and homozygous Cx26 cKO mice (Fig. 6d, $P=0.89$, one-way ANOVA). 
a

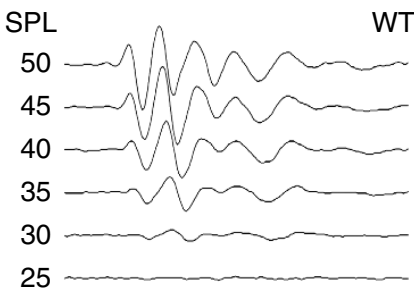

C

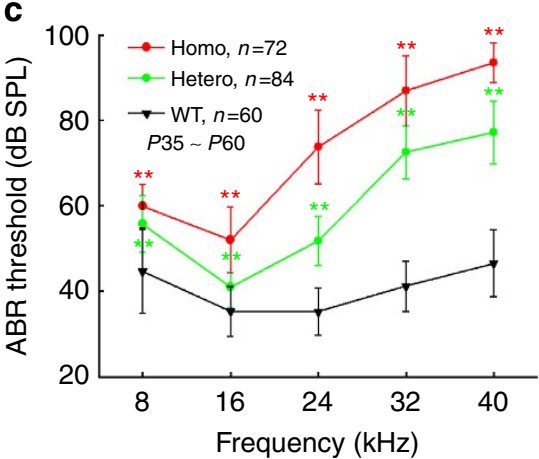

b

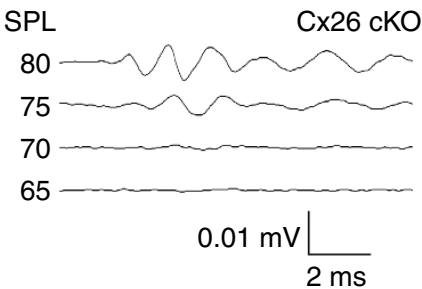

d

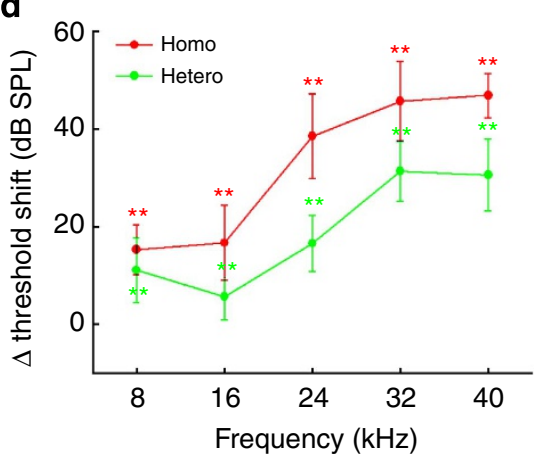

Figure 2 | Hearing loss in Cx26 cKO mice assessed by ABR recording. (a,b) ABR waveforms recorded in Cx26 cKO and WT mice. The ABR was evoked by 24-kHz tone-bursts at postnatal day 45 (P45). (c) Frequency thresholds of ABR in homozygous and heterozygous Cx26 cKO mice and WT mice at P35-60. WT littermates served as a control group. (d) Elevation of ABR thresholds in homozygous and heterozygous Cx26 cKO mice. Hearing loss is severe at high frequencies. Data are expressed as mean \pm s.d.; ${ }^{\star \star} P<0.001$ as determined by one-way ANOVA with a Bonferroni correction.
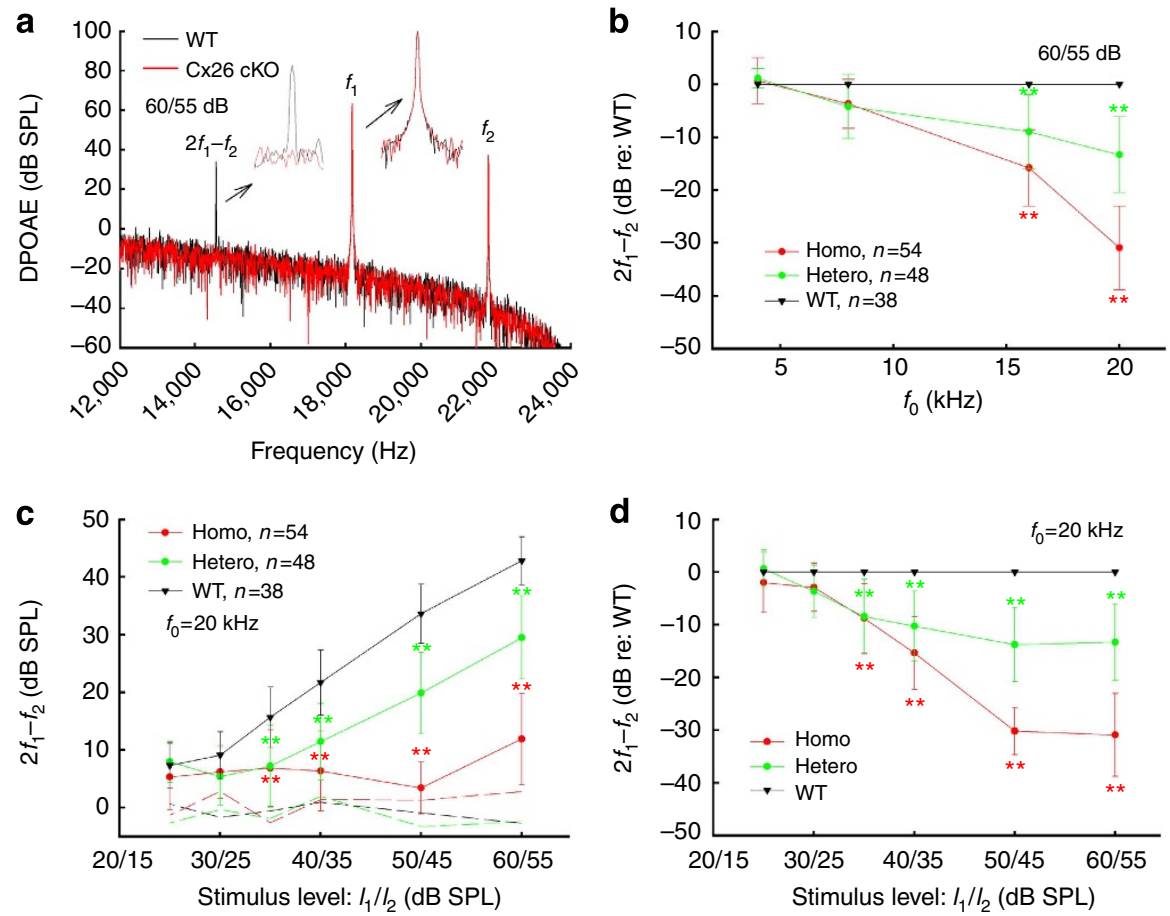

Figure 3 | Reduction of acoustic emission in Cx26 cKO mice. (a) Spectrum of acoustic emission recorded from Cx26 cKO mice and WT mice. Insets: large scale plotting of $2 f_{1}-f_{2}$ and $f_{1}$ peaks. The peak of DPOAE $\left(2 f_{1}-f_{2}\right)$ in Cx26 cKO mice was reduced but $f_{1}$ and $f_{2}$ peaks remained the same as those in WT mice. $f_{0}=20 \mathrm{kHz}$. (b) Reduction of DPOAE in Cx26 cKO mice in frequency responses. DPOAEs were normalized to the WT mice. (c) Reduction of DPOAE in Cx26 cKO mice in I/O plot. Dashed lines represent the noise levels of recording. (d) Normalized reduction of DPOAE in homozygous and heterozygous Cx26 cKO mice. Data are expressed as mean \pm s.d.; ${ }^{\star \star} P<0.001$ as determined by one-way ANOVA with a Bonferroni correction.

\section{Discussion}

We previously reported that in vitro uncoupling of gap junctions between DCs shifts OHC electromotility towards hyperpolarization and reduces the production of distortion products ${ }^{16}$, indicating that supporting cells can have a role in the regulation of OHC electromotility. In this study, we selectively deleted Cx26 expression in DCs and OPCs in vivo (Fig. 1 and Supplementary Fig. S1). We found that the deletion shifted OHC electromotility to the left in the same hyperpolarization direction (Figs 6 and 7) and caused DPOAE reduction and hearing loss 

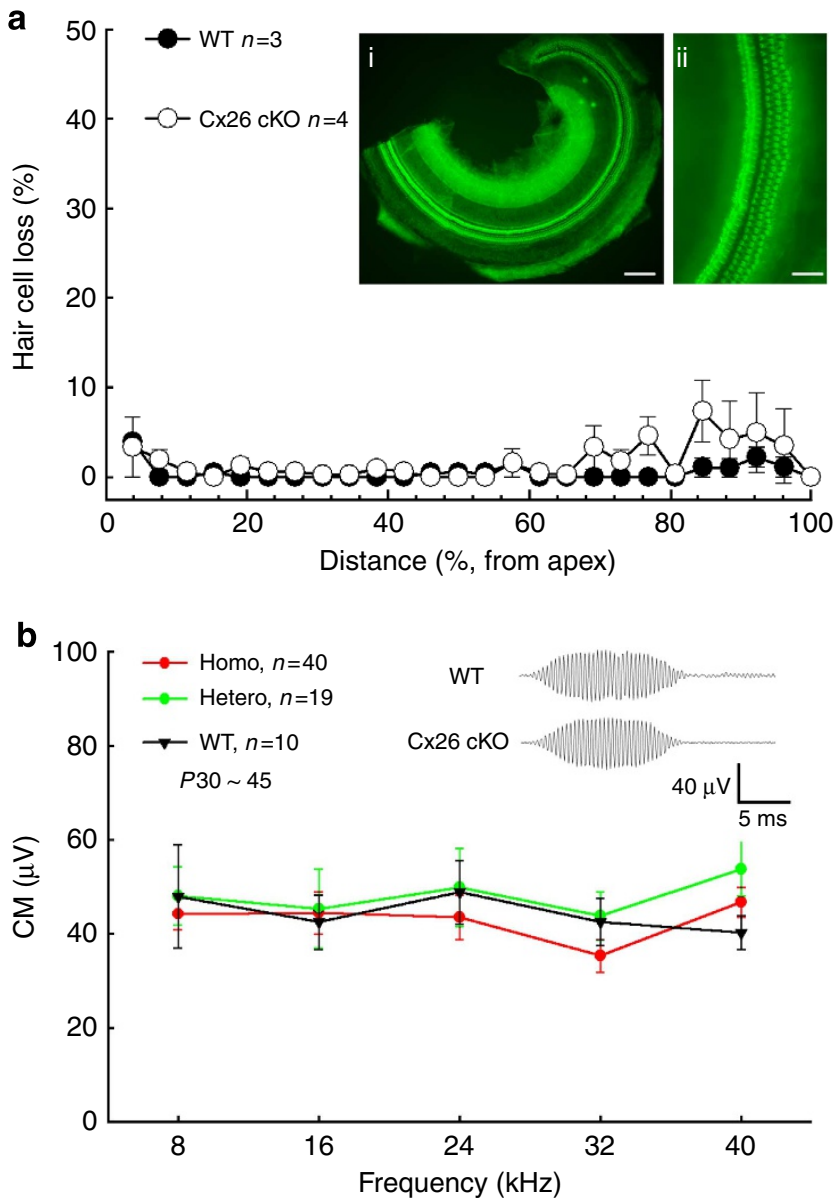

Figure 4 | Cx26 cKO mice have normal CM and no substantial hair cell loss. (a) Hair cell accounting at Cx26 cKO mice. Mice were P30-60 old. WT littermates served as control. Insets I and II: the whole-mounting cochlear sensory epithelium was stained by phalloidin for hair cell accounting. Scale bars, $100 \mu \mathrm{m}$ in I; $50 \mu \mathrm{m}$ in II. (b) CM responses of homozygous and heterozygous $\mathrm{C} \times 26$ cKO mice and WT mice. The CM was evoked by $90 \mathrm{~dB}$ SPL tone bursts. Insets: CM waveform recorded from WT and $\mathrm{C} \times 26$ cKO mice. Error bars represent s.d.

(Figs 2 and 3). These new findings provide direct evidence that cochlear supporting cells and gap junctions in vivo can regulate $\mathrm{OHC}$ electromotility to control active cochlear mechanics and hearing sensitivity.

Two active cochlear mechanics have been proposed: one is the prestin-based $\mathrm{OHC}$ electromotility ${ }^{1,3-5}$ and another is the stereocilium-based hair bundle movement $t^{2,27-29}$. Deletion of Cx26 in DCs and OPCs shifted OHC electromotility towards hyperpolarization (Figs 6 and 7). As previously reported ${ }^{30}$, this shift can reduce the gain of $\mathrm{OHC}$ electromotility amplification. The left-shift can also alter the phase difference between $\mathrm{OHC}$ electromotility and active force generation ${ }^{31,32}$. To augment sound-evoked vibrations of the $\mathrm{BM}$ in vivo, maximal $\mathrm{OHC}$ contraction need coincide with maximal $\mathrm{BM}$ velocity in the direction of scala vestibule 33,34 . Other timing relationships will cause smaller amplification or even attenuation. Indeed, DPOAE was significantly reduced in $\mathrm{Cx} 26 \mathrm{cKO}$ mice (Fig. 3), even prestin expression was not reduced (Figs 6c,d and 8). Recently, we found that Cx26 deficiency can alter supporting cell micromechanical property ${ }^{35}$. Supporting cell mechanical property change can alter $\mathrm{OHC}$ loading and membrane tension to shift $\mathrm{OHC}$ electromotility ${ }^{36,37}$ and the phase between the OHC length change and the feedback of active force to the BM vibration ${ }^{31,32}$. a
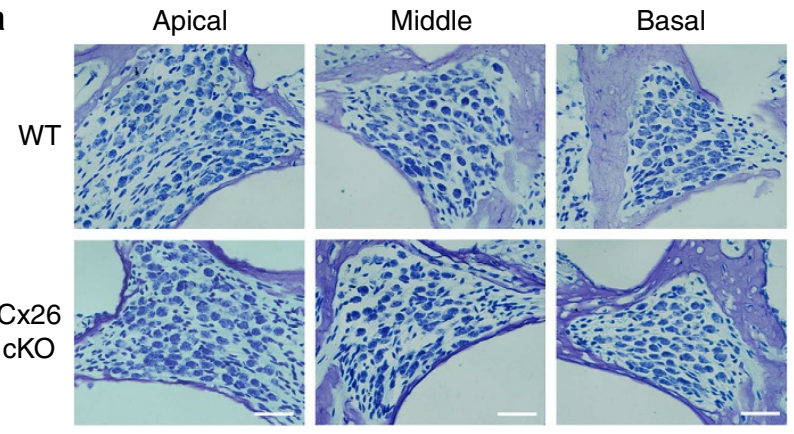

b

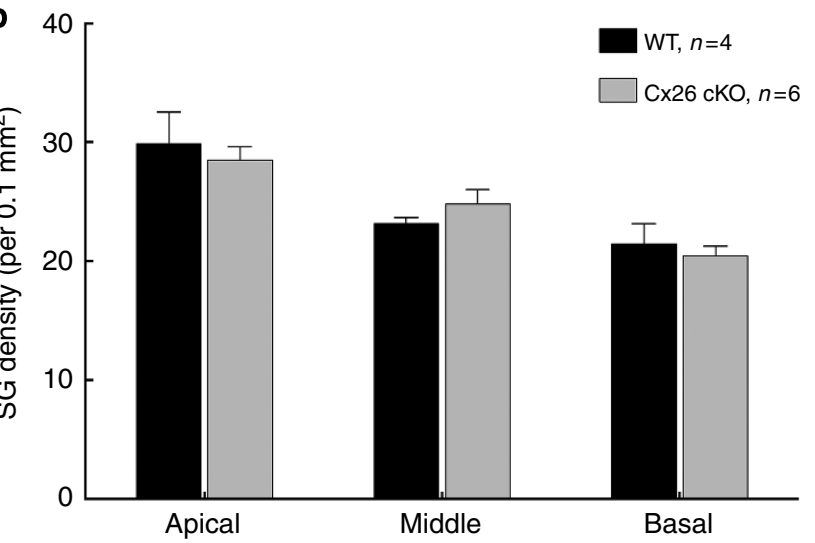

Figure 5 | No SG neuron degeneration in Cx26 cKO mice. (a) SGs in Rosenthal's canal at the apical, middle and basal turn. The sections were stained with toluidine blue. WT littermates served as control. The mice were P30-60 old. Scale bars, $30 \mu \mathrm{m}$. (b) The density of SGs in Rosenthal's canal at the apical, middle and basal turns. There is no significant difference in SG densities between Cx26 cKO mice and WT mice $(P>0.05$, one-way ANOVA). Data are expressed as mean \pm s.d.

As discussed above, these changes can eventually reduce the outcome of OHC electromotility and active cochlear amplification to induce hearing loss.

In $\mathrm{Cx} 26 \mathrm{cKO}$ mice, DPOAE reduction and hearing loss were larger and more severe at high frequencies (Figs 2 and 3 ). However, Cx26 deletion in DCs and OPCs was uniform at the apical, middle and basal turns (Supplementary Fig. S1). We previously found that Cx26 expression at the cochlear sensory epithelium is gradually reduced from the cochlear apex to base $\mathrm{e}^{14}$. The basal turn has less Cx26 expression. The reduction is more pronounced in DCs and OPCs. So, targeted-deletion of Cx26 in DCs and OPCs may produce more severe function impairment at high frequencies. In addition, deletion of Cx26 in DCs and OPCs can reduce gap junctional coupling and may impair potassium recycling as well ${ }^{19,38}$. Computer modelling shows that this can attenuate $\mathrm{OHC}$ transmembrane potential to reduce the activity of $\mathrm{OHC}$ electromotility, in particular, at the high-frequency region $^{39}$. Finally, shorter OHCs had larger shift in OHC electromotility (Figs 6 and 7). These coincident changes strongly suggest that targeted-deletion of Cx26 in DCs and OPCs left-shifted $\mathrm{OHC}$ electromotility resulting into active cochlear mechanics reduction and hearing loss.

Frequency-dependent hearing loss (Fig. 2) is also inconsistent with the EP reduction induced whole-frequency deafness. Moreover, Cx26 cKO mice retained normal CM and EP (Fig. 4b), implying that the $\mathrm{Cx} 26 \mathrm{cKO}$ mice may retain normal hair cell transduction activity. These data further support the concept that targeted-deletion of Cx26 in DCs and OPCs causes OHC 
a

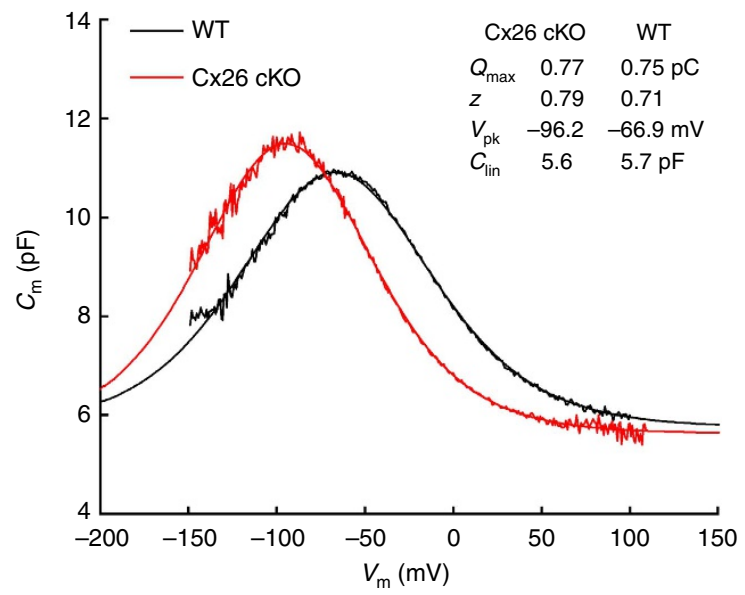

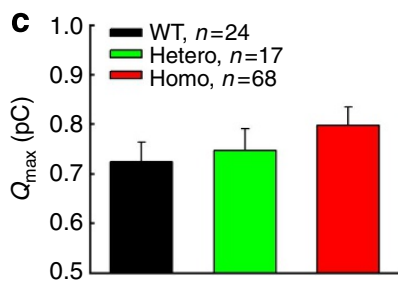

d
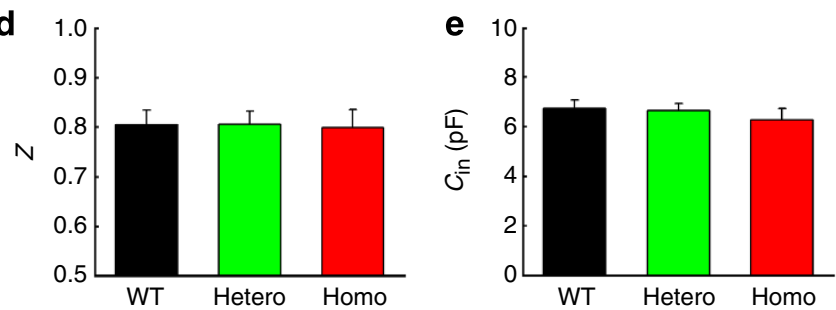

Figure 6 | Left-shift of OHC NLC in Cx26 cKO mice. (a) NLC recorded from $\mathrm{C} \times 26$ cKO and WT mice. Smooth lines represent fitting by the first derivative of Boltzmann equation. (b-e) Parameters of NLC fitting. WT littermates served as a control group. Mice were P50-60 old. ${ }^{\star *} P<0.001$ as determined by one-way ANOVA with a Bonferroni correction. Data are expressed as mean \pm s.d.

electromotility left-shifting rather than EP reduction to induce the reduction of active cochlear mechanics and hearing loss.

Cx26 mutations are a common genetic cause for nonsyndromic hearing loss ${ }^{18-20}$. However, the underlying deafness mechanisms still remain unclear. Previous mouse models with extensive deletion of Cx26 in the cochlea show that Cx26 deficiency can induce cochlear development disorder, hair cell degeneration and congenital deafness ${ }^{21,22,40}$. However, clinical phenotypes of Cx26 mutation are various. A large group $(\sim 30 \%)$ of $\mathrm{Cx} 26$ mutation patients show a progressive, late-onset hearing $\operatorname{loss}^{18-20}$. Our new study also found that hair cell degeneration is not a primary cause for $\mathrm{Cx} 26$ deficiency-induced hearing loss ${ }^{21}$. Some mechanisms other than cochlear development disorder and hair cell degeneration must be engaged. In this study, we found that targeted-deletion of Cx26 in DCs and OPCs had hearing loss (Fig. 2), but had no cochlear development disorders (Fig. 1 and Supplementary Fig. S1) and no significant hair cell and SG neuron degeneration (Figs 4 and 5). OHC electromotility was leftshifted (Figs 6 and 7) and DPOAE was reduced (Fig. 3). These new findings provide not only new information about supporting cell dependence of active cochlear mechanics in vivo but also a new mechanism for Cx26 deficiency-induced deafness, particularly, for late-onset hearing loss, which patients have a significant DPOAE reduction ${ }^{41,42}$.
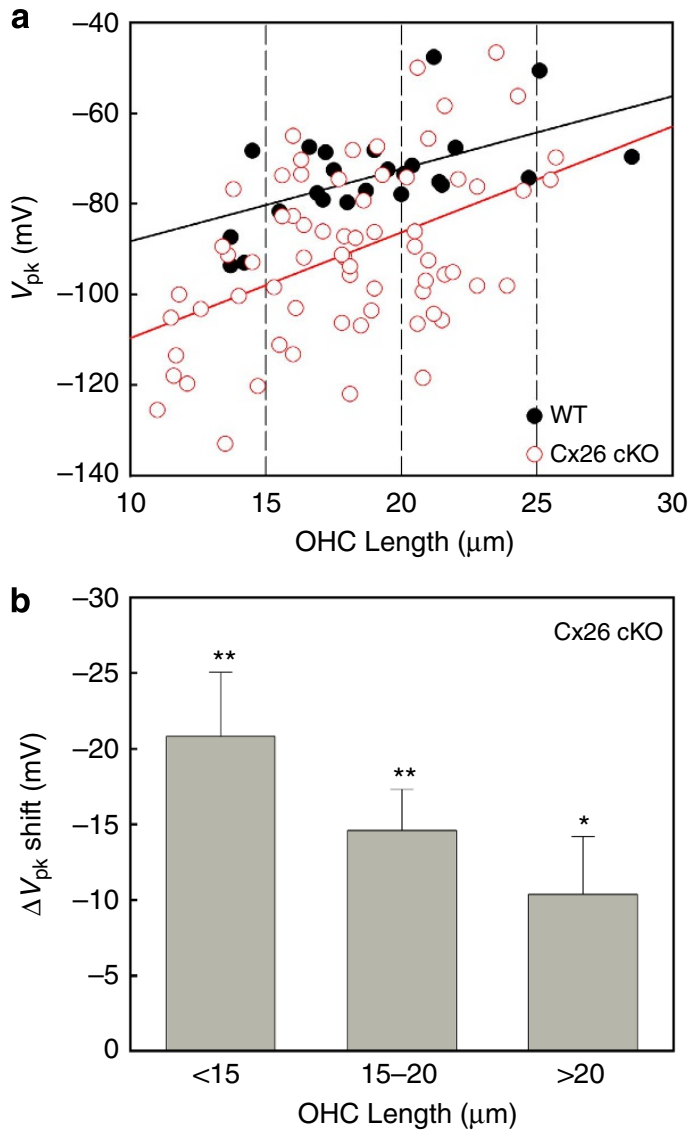

Figure 7 | Large-shifting of NLC in shorter OHCs. (a) Distribution of $V_{p k}$ of NLC with $\mathrm{OHC}$ length. The solid lines represent linear fitting: $y=1.60 x-104.3$ and $y=2.33 x-133.1$ for WT and Cx26 cKO mice, respectively. (b) $V_{p k}$ shifting in $\mathrm{C} \times 26 \mathrm{cKO}$ mice as a function of $\mathrm{OHC}$ length. In comparison with WT, changes of the $V_{p k}$ in $\mathrm{Cx} 26 \mathrm{cKO}$ mice were pooled in $\mathrm{OHC}$ length $<15,15-20,>20 \mu \mathrm{m}$ ranges and averaged. ${ }^{\star} P<0.05$, ${ }^{\star} * P<0.01$, one-way ANOVA with a Bonferroni correction. Data are expressed as mean \pm s.d.

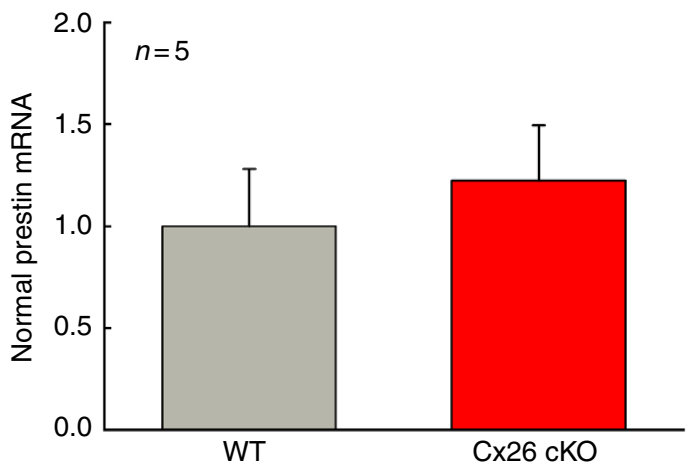

Figure 8 | No prestin expression reduction in Cx26 cKO mice. Prestin expression at the transcriptional level was measured by real-time RT-PCR. WT littermates served as controls. Prestin expression in the Cx26 cKO mice appears slightly increased rather than decreased. However, the increase is not significant ( $P=0.58$, one-way ANOVA). Data are expressed as mean \pm s.d.

\section{Methods}

Generation of Cx26 conditional KO mouse and genotyping. $C \times 26^{l o x P} / l o x P$ transgenic mice (EMMA, EM00245) ${ }^{22}$ were crossed with Prox1-CreER ${ }^{T 2}$ mouse line $^{23}$. Prox $1-C r e E R^{T 2} ; C x 26^{\operatorname{Lox} P(+/-)}$ mice were used for breeding. Tamoxifen 
(T5648, Sigma-Aldrich, St Louis, MO) was administrated to all litters at postnatal day 0 by intraperitoneal injection $(0.5 \mathrm{mg}$ per $10 \mathrm{~g} \times 3$ days $)$. Mouse genotyping was identified by PCR amplification of tail genomic $\mathrm{DNA}^{22,23}$. WT littermates were used as control. All experimental procedures were conducted in accordance with the policies of the University of Kentucky Animal Care \& Use Committee.

In vivo physiological measurements. $\mathrm{ABR}, \mathrm{DPOAE}$ and $\mathrm{CM}$ recordings were performed in a double-wall sound isolated chamber by use of a Tucker-Davis ABR \& DPOAE workstation with ES-1 high-frequency speaker (Tucker-Davis Tech., Alachua, FL ${ }^{21}$. Mice were anaesthetized by intraperitoneal injection with a mixture of ketamine and xylazine $(8.5 \mathrm{ml}$ saline $+1 \mathrm{ml}$ Ketamine $+0.55 \mathrm{ml}$ Xylazine, $0.1 \mathrm{ml}$ per $10 \mathrm{~g}$ ). Body temperature was maintained at $37-38^{\circ} \mathrm{C}$. ABR was measured by clicks in alternative polarity and tone bursts $(8-40 \mathrm{kHz})$ from 80 to $10 \mathrm{~dB}$ SPL in a $5-\mathrm{dB}$ step. The ABR threshold was determined by the lowest level at which an ABR can be recognized. If mice had severe hearing loss, the ABR test from 110 to $70 \mathrm{~dB}$ SPL was added. For CM recording, the electrode was ventrolaterally inserted deeply into the temporal bone ${ }^{21}$. The signal was amplified $(50,000 \times)$, filtered $(3-50 \mathrm{kHz})$ and averaged by 250 times.

For DPOAE recording, the test frequencies were presented by a geometric mean of $f_{1}$ and $f_{2}\left(f_{0}=\left(f_{1} \times f_{2}\right)^{1 / 2}\right)$ from $f_{0}=4-20 \mathrm{kHz}$ with a ratio of $f_{1}: f_{2}=1: 1.2$. The distortion product was recorded from the $L_{1} / L_{2}$ level of $60 / 55$ to $25 / 20 \mathrm{~dB}$ SPL with an average of 150 times. A cubic distortion component of $2 f_{1}-f_{2}$ was measured.

EP was measured by a lateral-wall access. Mice were anaesthetized and body temperature was maintained at $37-38^{\circ} \mathrm{C}$. The cochlea was exposed by a ventral approach. Access to the endolymphatic compartment (scala media) of the middle turn was gained by thinning the bone over the spiral ligament and picking a small hole. The glass microelectrode was filled with a $\mathrm{K}^{+}$-based intracellular solution (140 KCl, 10 EGTA, $2 \mathrm{MgCl}_{2}, 10$ HEPES (all in mM), pH7.2) and the EP was recorded with an AD/DA board (PCI-6052E, National Instruments, Austin, TX, USA).

Immunofluorescent staining and confocal microscopy. The cochlear tissue preparation and immunofluorescent staining were performed as previously reported $^{14}$. Monoclonal mouse anti-Cx26 (Cat. no. 33-5800, Invitrogen Corp.), polyclonal rabbit anti-Cx30 (Cat. no. 71-2200, Invitrogen Corp.) and polyclonal goat anti-prestin (Cat. no. sc-22694, Santa Cruz Biotech Inc., CA) were used and visualized by secondary Alexa Fluor 488- or 568-conjugated antibodies (Molecular Probes). The staining was observed under a confocal laser-scanning microscope.

Toluidine blue staining and SG neuron accounting. Consecutive cochlear paraffin sections were stained with toluidine blue using the conventional protocol ${ }^{21}$ To count the SG neurons, two neighbouring sections separated by $40 \mu \mathrm{m}$ were used to eliminate the possibility of double counting. Counting errors were corrected by Abercrombie's equation ${ }^{43}: T /(T+D)$, in which $T$ is the section thickness $(10 \mu \mathrm{m})$ and $D$ is the mean diameter $(8.65 \mu \mathrm{m})$ of the SG neurons. The area of Rosenthal's canal in the cochlear sections was measured by the NIH image software ${ }^{21}$. The densities of SG neurons in apical, middle and basal turns were calculated by the number of SG neurons in the section divided by the area of Rosenthal's canal.

Real-time RT-PCR measurement of prestin expression. As we previous reported $^{44}$, the cochlea was freshly isolated and homogenized. Its RNA was extracted by Absolutely RNA Miniprep Kit (Stratagene, La Jalla, CA), following the manufacturer's instructions. The quality and quantity of total mRNAs were determined by NanoDrop ND-1000 Spectrophotometer (NanoDrop Technologies, Inc., Rockland, DE). The obtained mRNA was converted to cDNA by iScript cDNA Synthesis Kit (Bio-Rad laboratories, Inc., Hercules, CA). Quantitative realtime PCR was performed by MyiQ real-time PCR detection system with iQ SYBR Green Super Mix kit (Cat. no.: 170822, Bio-Rad laboratories). The primers for prestin were $5^{\prime}$-GTT GGG TGG CAA GGA GTT TA-3', 5'-ACA GGG AGG ACA CAA AGG TG- $3^{\prime}$. The cycling condition of PCR amplification was $95^{\circ} \mathrm{C} 30 \mathrm{~s}, 55^{\circ} \mathrm{C}$ $30 \mathrm{~s}$, and $72{ }^{\circ} \mathrm{C} 30 \mathrm{~s}, 45$ cycles. Each cochlear sample was measured in triplicate and averaged. Universal 18S (QuantumRNATM 18S Internal Standards, Ambion Inc., Austin, TX) served as an internal control. The relative quantities of prestin expression were calculated from standard curves and normalized to the amount of $18 \mathrm{~S}$

Patch clamp recording of OHC nonlinear capacitance. OHCs were freshly isolated from the cochlea ${ }^{21,44}$. The classical patch clamp recording was performed under whole-cell configuration by using an Axopatch 200B patch clamp amplifier (Molecular Devices, CA) with jClamp (Scisft, New Haven, CT). NLC was measured by a two-sinusoidal method and fitted to the first derivative of a two-state Boltzmann function with jClamp and MATLAB ${ }^{16,44}$

$$
C_{\mathrm{m}}=N L C+C_{\text {lin }}=Q_{\max } \frac{z e}{k T} \frac{\exp \left(\frac{-z e\left(V_{\mathrm{m}}-V_{\mathrm{pk}}\right)}{k T}\right)}{\left(1+\exp \left(\frac{-z e\left(V_{\mathrm{m}}-V_{\mathrm{pk}}\right)}{k T}\right)\right)^{2}}+C_{\text {lin }}
$$

where $Q_{\max }$ is the maximum charge transferred, $V_{\mathrm{pk}}$ is the peak of NLC, $z$ is the number of elementary charge $(e), k$ is Boltzmann's constant and $T$ is the absolute temperature. Membrane potential $\left(V_{\mathrm{m}}\right)$ was corrected for electrode access resistance $\left(R_{\mathrm{s}}\right)$

Statistical analysis. Data were plotted by SigmaPlot and statistically analysed by SPSS v13.0 (SPSS Inc., Chicago, IL). Error bars represent s.e.m. other than indicated in text.

\section{References}

1. Dallos, P. Cochlear amplification, outer hair cells and prestin. Curr. Opin. Neurobiol. 18, 370-376 (2008).

2. Hudspeth, A. J. Making an effort to listen: mechanical amplification in the ear. Neuron 59, 530-545 (2008).

3. Brownell, W. E., Bader, C. R., Bertrand, D. \& Ribaupierre, Y. Evoked mechanical responses of isolated cochlear outer hair cells. Science 227, 194-196 (1985).

4. Zheng, J. et al. Prestin is the motor protein of cochlear outer hair cells. Nature 405, 149-155 (2000).

5. Ashmore, J. Cochlear outer hair cell motility. Physiol. Rev. 88, 173-210 (2008)

6. Zhao, H. B. \& Santos-Sacchi, J. Auditory collusion and a coupled couple of outer hair cells. Nature 399, 359-362 (1999).

7. Liberman, M. C. et al. Prestin is required for electromotility of the outer hair cell and for the cochlear amplifier. Nature 419, 300-304 (2002).

8. Slepecky, N. B. in The cochlea (Dallos, P., Popper, A. N. \& Fay, R. R. eds). 44-129 (Springer, 1996).

9. Parsa, A., Webster, P. \& Kalinec, F. Deiters cells tread a narrow path-The Deiters cells-basilar membrane junction. Hear. Res. 290, 13-20 (2012).

10. Dulon, D., Blanchet, C. \& Laffon, E. Photo-released intracellular $\mathrm{Ca}^{2+}$ evokes reversible mechanical responses in supporting cells of the guinea-pig organ of Corti. Biochem. Biophys. Res. Commun. 201, 1263-1269 (1994).

11. Bobbin, R. P. ATP-induced movement of the stalks of isolated cochlear Deiters cells. Neuroreport 12, 2923-2926 (2001).

12. Bobbin, P. P., Campbell, J., Lousteau, S. \& Mandhare, M. Electrical and ATP induced movements of the phalangeal processes of isolated cochlear Deiters' cells. In: Hair cell micromechanics and otoacoustic emissions (Berlin, C. I., Hood, L. J. \& Ricci, A. eds). 91-106 (Thomson Delmar Learning, 2002).

13. Kikuchi, T., Kimura, R. S., Paul, D. L. \& Adams, J. C. Gap junctions in the rat cochlea: immunohistochemical and ultrastructural analysis. Anat. Embryol. 191, 101-118 (1995).

14. Zhao, H. B. \& Yu, N. Distinct and gradient distributions of connexin26 and connexin30 in the cochlear sensory epithelium of guinea pigs. J. Comp. Neurol. 499, 506-518 (2006).

15. Zhao, H. B. Directional rectification of gap junctional voltage gating between Deiters cells in the inner ear of guinea pig. Neurosci. Lett. 296, 105-108 (2000)

16. Yu, N. \& Zhao, H. B. Modulation of outer hair cell electromotility by cochlear supporting cells and gap junctions. PLoS ONE 4, e7923 (2009).

17. Forge, A. et al. Gap junctions in the inner ear: comparison of distribution patterns in different vertebrates and assessement of connexin composition in mammals. J. Comp. Neurol. 467, 207-231 (2003).

18. Kelsell, D. P. et al. Connexin 26 mutations in hereditary non-syndromic sensorineural deafness. Nature 387, 80-83 (1997).

19. Zhao, H. B., Kikuchi, T., Ngezahayo, A. \& White, T. W. Gap junctions and cochlear homeostasis. J. Memb. Biol. 209, 177-186 (2006).

20. Castillo, F. J. \& Castillo, I. The DFNB1 subtype of autosomal recessive nonsyndromic hearing impairment. Front. Biosci. 17, 3252-3274 (2011).

21. Liang, C., Zhu, Y., Zong, L., Lu, G. J. \& Zhao, H. B. Cell degeneration is not a primary causer for Connexin26 (GJB2) deficiency associated hearing loss. Neurosci. Lett. 528, 36-41 (2012).

22. Cohen-Salmon, M. et al. Targeted ablation of connexin26 in the inner ear epithelial gap junction network causes hearing impairment and cell death. Curr. Biol. 12, 1106-1111 (2002).

23. Srinivasan, R. S. et al. Lineage tracing demonstrates the venous origin of the mammalian lymphatic vasculature. Genes Dev. 21, 2422-2432 (2007).

24. Bermingham-McDonogh, O. et al. Expression of Prox1 during mouse cochlear development. J. Comp. Neurol. 496, 172-186 (2006).

25. Kirjavainen, A. et al. Proxl interacts with Atoh1 and Gfil, and regulates cellular differentiation in the inner ear sensory epithelia. Dev. Biol. 322, 33-45 (2008).

26. $\mathrm{Yu}, \mathrm{Y}$. et al. In vivo proliferation of postmitotic cochlear supporting cells by acute ablation of the retinoblastoma protein in neonatal mice. J. Neurosci. 30, 5927-5936 (2010).

27. Chan, D. K. \& Hudspeth, A. J. $\mathrm{Ca}^{2+}$ current-driven nonlinear amplification by the mammalian cochlea in vitro. Nat. Neurosci. 8, 149-155 (2005).

28. Kennedy, H. J., Crawford, A. C. \& Fettiplace, R. Force generation by mammalian hair bundles supports a role in cochlear amplification. Nature 433, 880-883 (2005).

29. Fettiplace, R. Active hair bundle movements in auditory hair cells. J. Physiol. 576, 29-36 (2006).

30. Rajagopalan, L. et al. Tuning of the outer hair cell motor by membrane cholesterol. J. Biol. Chem. 282, 36659-36670 (2007). 
31. Fridberger, A., Flock, A., Ulfendahl, M. \& Flock, B. Acoustic overstimulation increases outer hair cell $\mathrm{Ca}^{2}+$ concentrations and causes dynamic contractions of the hearing organ. Proc. Natl Acad. Sci. USA 95, 7127-7132 (1998).

32. Flock, A., Flock, B., Fridberger, A., Scarfone, E. \& Ulfendahl, M. Supporting cells contribute to control of hearing sensitivity. J. Neurosci. 19, 4498-4507 (1999).

33. Gummer, A. W., Hemmert, W. \& Zenner, H. P. Resonant tectorial membrane motion in the inner ear: its crucial role in frequency tuning. Proc. Natl Acad. Sci. USA 93, 8727-8732 (1996).

34. Zha, D. et al. In vivo outer hair cell length changes expose the active process in the cochlea. PLoS ONE 7, e32757 (2012).

35. Chen, J. et al. New function for Connexin26 (Cx26) in the cochlea and new mechanism for deafness induced by deficiency in Cx26. The 36th Assoc. Res. Otolaryngol. Annual Meeting. Baltimore, MD, http://www.aro.org (2013).

36. Iwasa, K. H. Effect of stress on the membrane capacitance of the auditory outer hair cell. Biophys. J. 65, 492-498 (1993).

37. Kakehata, S. \& Santos-Sacchi, J. Membrane tension directly shifts voltage dependence of outer hair cell motility and associated gating charge. Biophys. J. 68, 2190-2197 (1995).

38. Zhu, Y. \& Zhao, H. B. ATP-mediated potassium recycling in the cochlear supporting cells. Purinergic Signal. 6, 221-229 (2010).

39. Mistrík, P. \& Ashmore, J. F. Reduced electromotility of outer hair cells associated with connexin-related forms of deafness: an in silico study of a cochlear network mechanism. J. Assoc. Res. Otolaryngol. 11, 559-571 (2010).

40. Wang, Y. et al. Targeted connexin 26 ablation arrests postnatal development of the organ of Corti. Biochem. Biophys. Res. Commun. 385, 33-37 (2009).

41. Engel-Yeger, B. et al. The effects of a connexin 26 mutation-35delG-on otoacoustic emissions and brainstem evoked potentials: homozygotes and carriers. Hear Res. 163, 93-100 (2002).
42. Engel-Yeger, B. et al. Otoacoustic emissions and brainstem evoked potentials in compound carriers of connexin 26 mutations. Hear Res. 175, 140-151 (2003).

43. Guillery, R. W. On counting and counting errors. J. Comp. Neurol. 447, 1-7 (2002).

44. Yu, N. et al. Prestin up-regulation in chronic salicylate (aspirin) administration: An implication of functional dependence of prestin expression. Cell Mol. Life Sci. 65, 2407-2418 (2008).

\section{Acknowledgements}

We thank Dr Guillermo Oliver at St Jude Children's Research Hospital, Memphis, TN for kindly providing Prox1-Cre mouse line. This work was supported by NIH R01 DC 05989.

\section{Author contributions}

Y.Z., C.L., L.Z., J.C. and H.B.Z. conducted the experiments and analysed the data. G.D.C contributed to Fig. 4a. H.B.Z. wrote the paper.

\section{Additional information}

Supplementary Information accompanies this paper at http://www.nature.com/ naturecommunications

Competing financial interests: The authors declare no competing financial interests.

Reprints and permission information is available online at http://npg.nature.com/ reprintsandpermissions/

How to cite this article: Zhu, Y. et al. Active cochlear amplification is dependent on supporting cell gap junctions. Nat. Commun. 4:1786 doi: 10.1038/ncomms2806 (2013) 